\title{
Long-time behavior of orbital periods of some Algol-type eclipsing binaries $^{\star}$
}

\author{
Qian Shengbang ${ }^{1,2,3}$ \\ 1 Yunnan Observatory, Chinese Academy of Sciences, P.O. Box 110, 650011 Kunming, PR China \\ e-mail: qsb@netease.com \\ 2 National Astronomical Observatories, Chinese Academy of Science (NAOCAS), PR China \\ 3 United Laboratory of Optical Astronomy, Chinese Academy of Science (ULOAC), PR China
}

Received May 2; accepted July 19, 2000

\begin{abstract}
Orbital period variations of six semi-detached binaries, UW Cyg, RX Hya, AK Ser, AC Tau, UW Vir and $\mathrm{VV} \mathrm{Vul}$, are presented based on the analysis of their O-C observations. For AK Ser and VV Vul, their orbital periods may show secular increase at rates of $\mathrm{d} P / \mathrm{d} t=$ $+7.9410^{-7}$ and $+3.4310^{-6}$ days/year. For UW Cyg, its orbital period is revised, and the $\mathrm{O}-\mathrm{C}$ curve shows a cyclic variation with a period of 49.2 years and an amplitude of 0.0383. For the other three systems, RX Hya, AC Tau and UW Vir, the changes in their orbital periods appear more complex but their $\mathrm{O}-\mathrm{C}$ curves can be represented by periodic variations (with periods of 54.3, 29.8 and 45.9 years) superimposed on upward parabolic segments. The components of upward curving parabolic variations in RX Hya, AC Tau and UW Vir indicate secular period increase with rates of $\mathrm{d} P / \mathrm{d} t=+1.0410^{-6},+7.1110^{-7}$ and $+1.7310^{-6}$ days/year respectively. The secular period increase in the five systems, RX Hya, AK Ser, AC Tau, UW Vir and VV Vul, can be explained by mass transfer from the less to the massive components, which is consistent with their semi-detached configurations. The periodic changes of the orbital periods in UW Cyg, RX Hya, AC Tau, UW Vir may be caused by the light-time effects due to the existence of the third bodies.
\end{abstract}

Key words: stars: individuals: UW Cyg, RX Hya, AK Ser, AC Tau, UW Vir and VV Vul

\section{Introduction}

The analysis of the orbital period variations of eclipsing binaries is very useful for the study of many physical

Send offprint requests to: Qian Shengbang

* Tables 2 to 7 are only available in electronic form at http://www.edpsciences.org processes. It provides estimates of mass transfer and loss, angular momentum transfer and loss, stellar evolution, third body problem, etc. In this paper, the long-time variations in the orbital periods of some Algol-type binaries, UW Cyg, RX Hya, AK Ser, AC Tau, UW Vir and VV Vul, are analyzed. Then the mechanisms that could be at the origin of the changes in the orbital periods are discussed.

The light variation of RX Hya was discovered by Miss H.S. Leavitt (Pickering 1907). After its discovery, the system was observed visually by many authors (e.g., Zinner 1922; Maggini 1921; Dugan 1933 and others). The first radial-velocity curve for the single-line binary system was published by Struve (1946) and the spectroscopic elements were also determined by him. Vyas \& Abhyankar (1989) obtained the first complete photoelectric $U, B$ and $V$ light curves and analyzed these observations with WilsonDevinney (W-D) method. Their studies suggest that RX Hya is a semi-detached Algol type binary with the secondary filling its Roche Lobe. By combining the spectroscopic elements from Struve (1946), the absolute parameters of the system are determined.

According to General Catalogue of Variable Stars (Kholopov 1985), the other five systems, UW Cyg, AK Ser, AC Tau, UW Vir and VV Vul are also semidetached binaries with orbital periods less than four days. The general properties of the five systems and those of RX Hya are shown in Table 1. Up to now, neither photoelectric or CCD light curves nor radial velocity curves of the stars have been published. Although ephemerides of these eclipsing binaries have been given by several authors (Mallama 1980; Wood \& Forbes 1963; Whitney 1959), they are neglected for period studies.

\section{Variation in the orbital period of UW Cygni}

For the period study of UW Cyg, all available times of light minimum have been compiled. 18 of the timings 
Table 1. General properties of Six eclipsing binaries

\begin{tabular}{llcccccc}
\hline Stars & Sp. & $P$ & $M_{1}$ & $R_{1}$ & $M_{2}$ & $R_{2}$ & Ref. \\
\hline UW Cyg & F0 & 3.45 & 1.71 & 2.28 & 0.59 & 2.79 & $(1)$ \\
RX Hya & F1+G5IV & 2.28 & 2.72 & 2.17 & 0.66 & 2.93 & $(2)$ \\
AK Ser & A5 & 1.92 & 1.98 & 1.97 & 0.93 & 2.12 & $(1)$ \\
AC Tau & F0 & 2.04 & 1.45 & 2.30 & 0.99 & 2.90 & $(1)$ \\
UW Vir & A2+k3IV & 1.81 & 1.77 & 1.52 & 0.43 & 2.08 & $(1)$ \\
VV Vul & A2/3V & 3.41 & - & - & - & - & $(3)$ \\
\hline
\end{tabular}

References in Table 1:

(1) Brancewicz \& Dworak (1980); (2) Vyas \& Abhyankar (1989); (3) Kholopov (1985).

published before 1959 have been collected and tabulated in the paper of Whitney (1959). Many times of light minimum are obtained from the Eclipsing Minimum Database by Internet (www.oa.uj.edu.pl/ktt/ktt.html). In all, 112 timings are collected. Unfortunately, all of the timings are visual or photographic. In this section, with these times of light minimum, the change in the orbital period of the neglected system is studied. In order to see the general behavior of the variations in the period of the system, the $\mathrm{O}-\mathrm{C}$ residuals of all times of light minimum were computed by the linear ephemeris given in the fourth edition of the "General Catalogue of Variable Stars", as

MinI $=2443690.0355+3.4507805 \times E$

and listed as $(\mathrm{O}-\mathrm{C})_{1}$ in Table 2 . The $(\mathrm{O}-\mathrm{C})_{1}$ values are presented graphically against epoch number in Fig. 1. During the calculation, times of light minimum with the same epoch have been averaged and some timings listed in the first and sixth columns are the mean values. Two photographic times of light minimum 2445201.434 and 49166.000 are discarded, because their O-C values show large deviations from the general $\mathrm{O}-\mathrm{C}$ trend formed by other points.

Since the form of the $\mathrm{O}-\mathrm{C}$ diagram depends very much on the choice of the ephemeris used in the calculation of the "C" epochs, the general trend of the $(\mathrm{O}-\mathrm{C})_{1}$ diagram may show a downward straight line, indicating that this ephemeris need to be revised. Based on all times of light minimum, a least-squares solution yields the following mean ephemeris:

$\operatorname{MinI}=2443690.0670(31)+3.4507620(13) \times E$.

The residuals $(\mathrm{O}-\mathrm{C})_{2}$, calculated with this ephemeris, are listed in fifth and tenth columns of Table 2 and are plotted graphically against epoch number in Fig. 2, from which the following results can be drawn: (i) the orbital period of the system is variable; (ii) its variation may show a wavelike fluctuation. With least-square method, a periodic ephemeris is obtained, as:

$(\mathrm{O}-\mathrm{C})_{2}=-0.0055+0.0383 \sin \left(0^{\circ} 0691 \times E+147.1^{\circ}\right)(3)$ which can give a good fit to the general trend of the $(\mathrm{O}-\mathrm{C})_{2}$ values (solid line in Fig. 2). This formula clearly indicates a periodic variation with a period of about

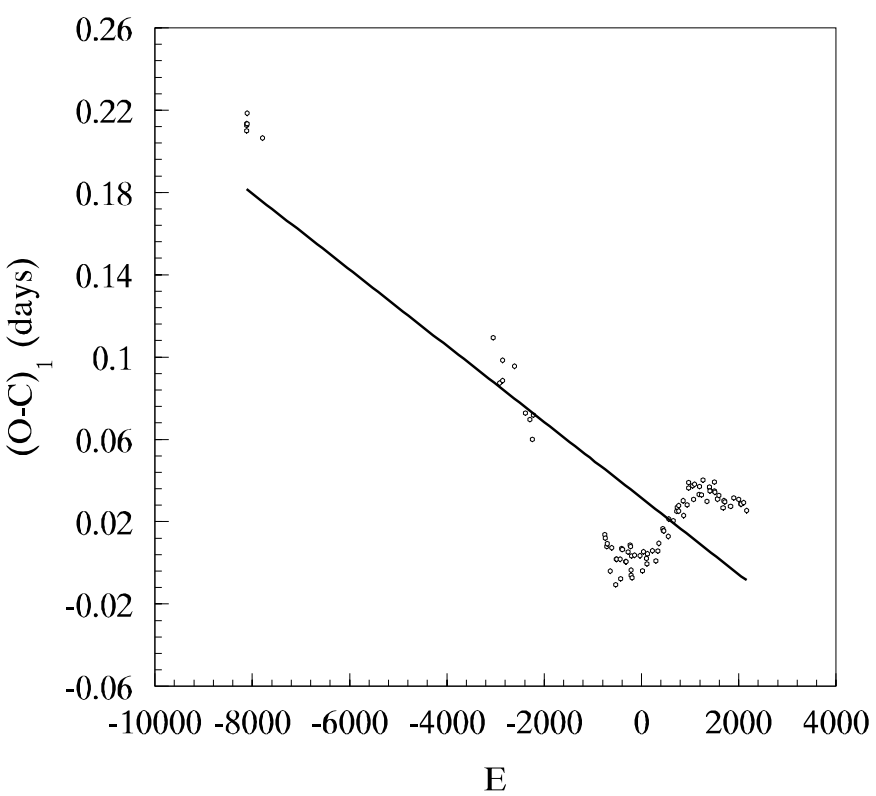

Fig. 1. The O-C curve of UW Cyg from the ephemeris given by GCVS. The solid line refers to a new ephemeris

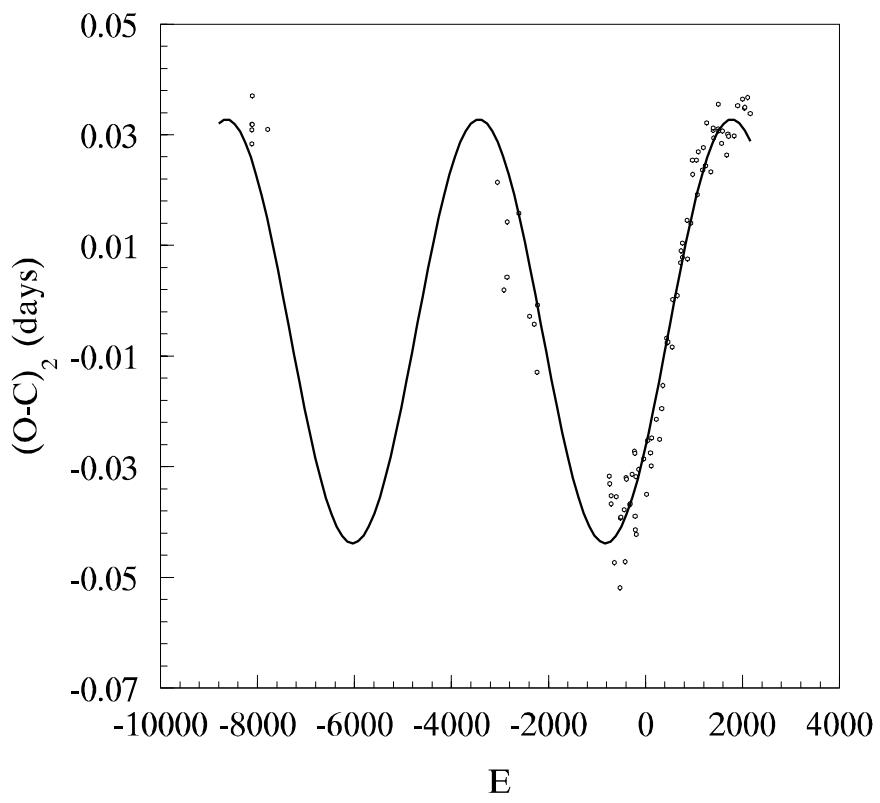

Fig. 2. O-C residuals based on the new ephemeris. The solid line refers to its description by a periodic function

$T=49.2$ year and an amplitude of about $A=0.0383$. The cyclic variation of the $\mathrm{O}-\mathrm{C}$ curve strongly suggests that the change of the orbital period is periodic.

\section{Variation in the orbital period of RX Hya}

The orbital period of RX Hya has been studied by Vyas \& Abhyankar (1989). They collected 6 photoelectric and 55 visual times of light minimum and pointed out that the $\mathrm{O}-\mathrm{C}$ curve showed a cyclic variation with a period of 72.46 years and an amplitude of 0.069 . By omitting 


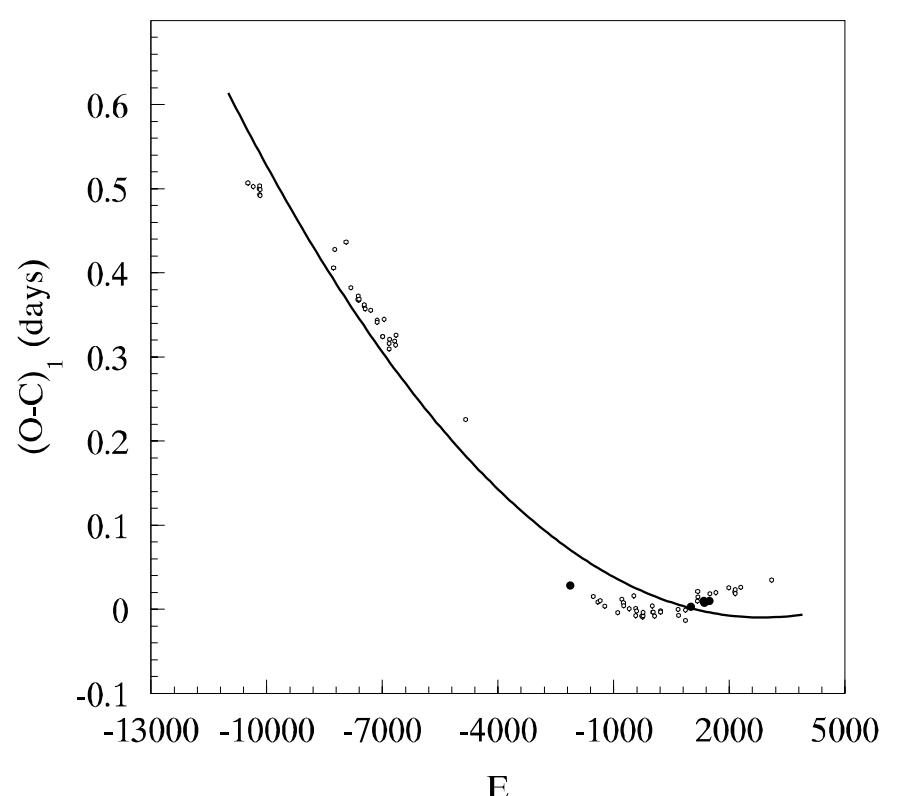

Fig. 3. O-C diagram of RX Hydrae. Circles refer to visual and photographic, and dots to photoelectric observations. Also given in solid line is the description of the general trend of the O-C curve

a few visual timings of earlier epoch, they obtained a rather large increase rate $\left(\mathrm{d} P / \mathrm{d} t=1.0210^{-5}\right.$ days/year $)$ for the change of the orbital period. After Vyas and Abhyankar's study, many times of light minimum have been collected at Eclipsing Minimum Database. With these timings, the changes in the orbital period of the system are analyzed. In order to study the variations in the period of the system, the residuals $(\mathrm{O}-\mathrm{C})_{1}$ based on the following ephemeris:

MinI $=2443447.700+2 \mathrm{~d} 2816450 \times E$

given in GCVS, are calculated. These $(\mathrm{O}-\mathrm{C})_{1}$ values are listed in the fifth and ninth columns of Table 3 and shown graphically against epoch number in Fig. 3. During the calculation, timings with the same epoch have been averaged and some times of light minimum listed in Table 3 are the mean values.

As displayed in Fig. 3, the orbital period of the system varies in some complex ways. Since the general trend of the $(\mathrm{O}-\mathrm{C})_{1}$ diagram may show a roughly parabolic distribution indicating a long-time increase in the orbital period, with the weight 3 for visual and photographic, and 8 for photoelectric times of light minimum, a second-order least-squares solution of the $(\mathrm{O}-\mathrm{C})_{1}$ values yields the following ephemeris:

$$
\begin{gathered}
\text { MinI }=2443447.7165+2.28162647 \times E+3.25 \quad 10^{-9} \times E^{2} \\
\pm 82 \quad \pm 15 \quad \pm 2
\end{gathered}
$$

where the coefficient of the square term represents the rate of change of the period and a continuous period increase of $\mathrm{d} P / \mathrm{d} E=+6.510^{-9}$ days $/$ cycle $=+1.0410^{-6}$ days $/$ year

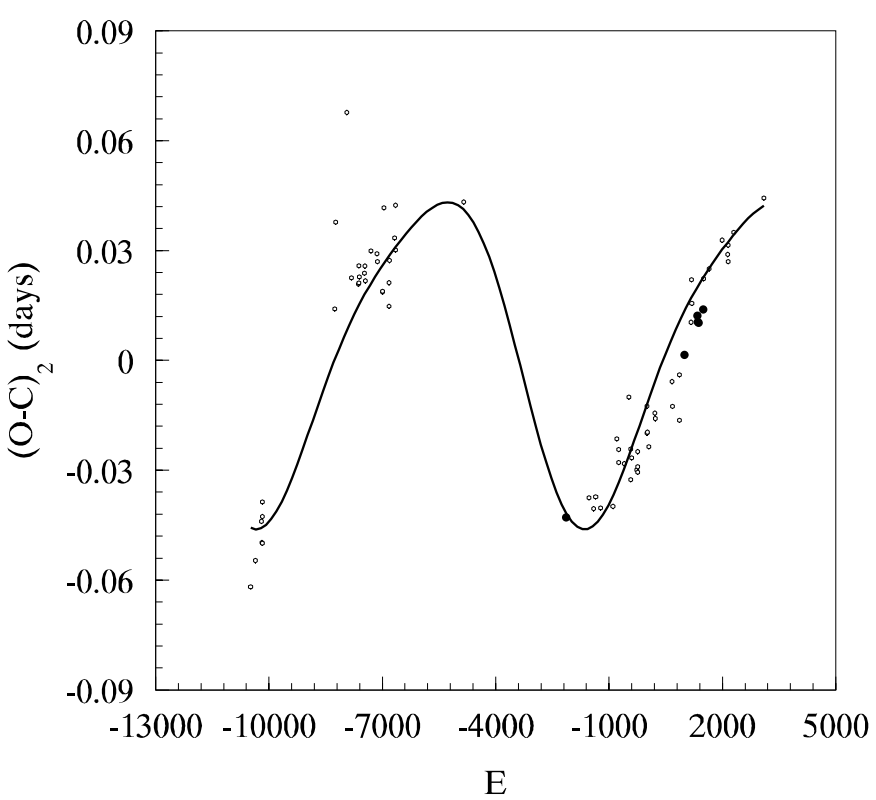

Fig. 4. O-C residuals of RX Hydrae based on the quadratic ephemeris (5). Solid line is the fit of the third body solution

is calculated which is equivalent to a period increase of $9.0 \mathrm{~s} /$ century. The secular increase only indicates the general trend of the $(\mathrm{O}-\mathrm{C})_{1}$ diagram without describing any particular characteristics.

The $(\mathrm{O}-\mathrm{C})_{2}$ residuals from the quadratic ephemeris (5) are also listed in Table 3 and displayed in Fig. 4. The $(\mathrm{O}-\mathrm{C})_{2}$ values in Fig. 4 clearly show a periodic oscillation. Most of the observations are photographic or visual and all the times of light minimum are primary, this may be caused by the fact that the secondary is more difficult to observe by such methods. We do not know whether secondary times of light minimum follow the same trend of the $\mathrm{O}-\mathrm{C}$ variation. The circular orbit of RX Hya (Lucy \& Sweeney 1980) suggests that the periodic oscillation of the $(\mathrm{O}-\mathrm{C})_{2}$ residuals is not caused by apsidal motion and that the light-time effect caused by the presence of a third body may be the real reason. The not strictly sine-like variation of the $(\mathrm{O}-\mathrm{C})_{2}$ curve suggests that the third body is moving in an elliptical orbit. The following formula:

$(\mathrm{O}-\mathrm{C})_{2}=a_{0}+\sum_{i=1}^{2}\left[a_{i} \sin (i \omega E)+b_{i} \sin (i \omega E)\right]$

has been used to express these $(\mathrm{O}-\mathrm{C})_{2}$ values which are the result of various numerical trials with different expressions and give the best fitting with the observations (solid line in Fig. 4). By least-squares solution of equation (6), the following values are obtained: $a_{0}=0.0031$, $a_{1}=0.0369, b_{1}=-0.0224, a_{2}=0.0021, b_{2}=0.0073$ and $\omega=0.0414$. With $\omega=360^{\circ} P_{\mathrm{e}} / T$, the orbital period of the eclipsing binary around the centre of mass of the triple system is determined to be: $T=54.3$ years.

From a visual inspection of the trend of these $(\mathrm{O}-\mathrm{C})_{2}$ residuals, it is seen that the orbital eccentricity of the 
eclipsing binary around the common centre of the gravity of the three bodies is small, and the orbital parameters can be directly determined from the folowing formular (Kopal 1959):

$a_{12}^{\prime} \sin i^{\prime}=c \sqrt{a_{1}^{2}+b_{1}^{2}}$

$e^{\prime}=2 \sqrt{\frac{a_{2}^{2}+b_{2}^{2}}{a_{1}^{2}+b_{1}^{2}}}$

$\omega^{\prime}=\arctan \frac{\left(b_{1}^{2}-a_{1}^{2}\right) b_{2}+2 a_{1} a_{2} b_{1}}{\left(a_{1}^{2}-b_{1}^{2}\right) a_{2}+2 a_{1} b_{1} b_{2}}$

$\tau^{\prime}=t_{0}-\frac{P^{\prime}}{2 \pi} \arctan \frac{a_{1} b_{2}-b_{1} a_{2}}{a_{1} a_{2}+b_{1} b_{2}}$

where $c$ is the speed of light, $a_{12}^{\prime}$ is the semi-major axis of the eclipsing pair around the common centre of the gravity of the triple system, $i^{\prime}, e^{\prime}, w^{\prime}, \tau^{\prime}$ and $P^{\prime}$ are the usual elements of the third body orbit. The results are: $a_{12}^{\prime} \sin i^{\prime}=7.47 \mathrm{AU}, e^{\prime}=0.34, w^{\prime}=43.5^{\circ}$ and $\tau^{\prime}=2447568 \mathrm{~d} 5$.

\section{Variation in the orbital period of AK Ser}

AK Ser is an EA-type eclipsing binary with sectral type of A5. 32 visual and photographic times of light minimum published in BBSAG bulletin have been collected at Eclipsing Minimum Database and 1 timing 2436348.472 was published by Szczepanowska (1959). Using the linear ephemeris given in the fourth edition of the "General Catalogue of Variable Stars":

MinI $=2446255.462+1.922580 \times E$

the $\mathrm{O}-\mathrm{C}$ residuals of all times of light minimum were computed and listed in Table 4 . These $\mathrm{O}-\mathrm{C}$ values are presented in Fig. 5.

As displayed in Fig. 5, the orbital period of the system is variable. Based on all the times of light minimum, a least square solution yields the following quadratic ephemeris:

$\operatorname{MinI}=2446255.4624+1.92258268 \times E+2.0910^{-9} \times E^{2}(12)$

$$
\pm 3 \quad \pm 34 \quad \pm 8
$$

and a period increase rate of $\mathrm{d} P / \mathrm{d} t=+7.9410^{-7}$ days $/$ year. Since the time interval between the first timing and the other times of light minimum is large (about 4200 cycles or 22 years), if the first time of light minimum is not used in the least square solution, we can obtain another quadratic ephemeris:

$$
\begin{gathered}
\operatorname{MinI}=2446255.4631+1.92258273 \times E+1.7010^{-9} \times E^{2}(13) \\
\pm 135 \pm 80 \quad \pm 60
\end{gathered}
$$

and a period increase rate of $\mathrm{d} P / \mathrm{d} t=+6.4610^{-7}$ days $/$ year which is nearly equal to that value obtained by using all timings.

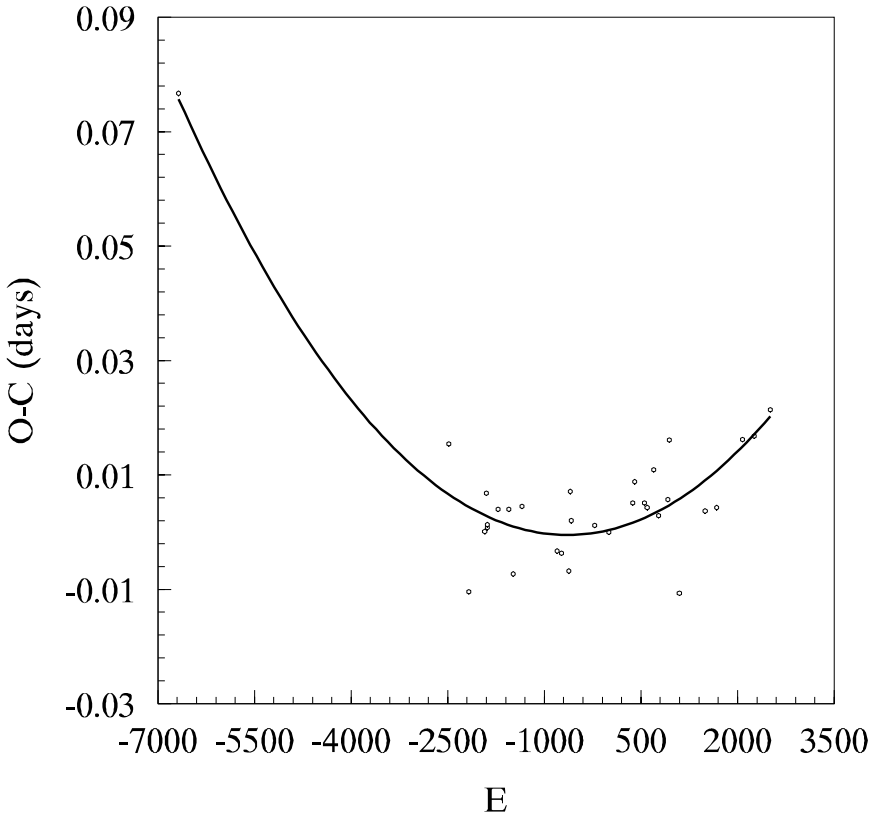

Fig. 5. O-C curve of AK Ser and its description by a quadratic ephemeris

\section{Variation in the orbital period of AC Tau}

In order to study the changes in the orbital period of $\mathrm{AC} \mathrm{Tau}$, various times of light minimum are compiled. Many visual and photographic times of light minimum observed by many amateurs have been collected at Eclipsing Minimum Database and 7 visual timings from Cracow observatory were published by Kreiner (1976). These timings are listed in the first and sixth columns of Table 5. Timings with the same epoch numbers have been averaged and some times of light minimum listed in Table 5 are the mean values of these timings. With the following ephemeris given in the fourth edition of GCVS:

MinI $=2445636.590+2.043356 \times E$

the residuals $(\mathrm{O}-\mathrm{C})_{1}$ of these timings are computed. These $(\mathrm{O}-\mathrm{C})_{1}$ valuse are listed in the fourth and ninth columns of Table 5 and are plotted in Fig. 6. One photographic minimum 2445057.522 shows large deviation from the general $\mathrm{O}-\mathrm{C}$ trend formed by other points in Fig. 6 . This point was not considered further in the discussion of the period variation.

As displayed in Fig. 6, we can conclude that the orbital period of the system is variable, and its variation is complex. Since the general trend of the $(\mathrm{O}-\mathrm{C})_{1}$ diagram may show a roughly parabolic distribution indicating a secular increase in the period, a least-squares solution of the $(\mathrm{O}-\mathrm{C})_{1}$ values yields the following ephemeris:

$\operatorname{MinI}=2445636.6018+2.04337351 \times E+1.9910^{-9} \times E^{2}(15)$

$$
\pm 14 \quad \pm 17 \quad \pm 2
$$

where the coefficient of the square term represents the rate of change of the period. This ephemeris can be used 


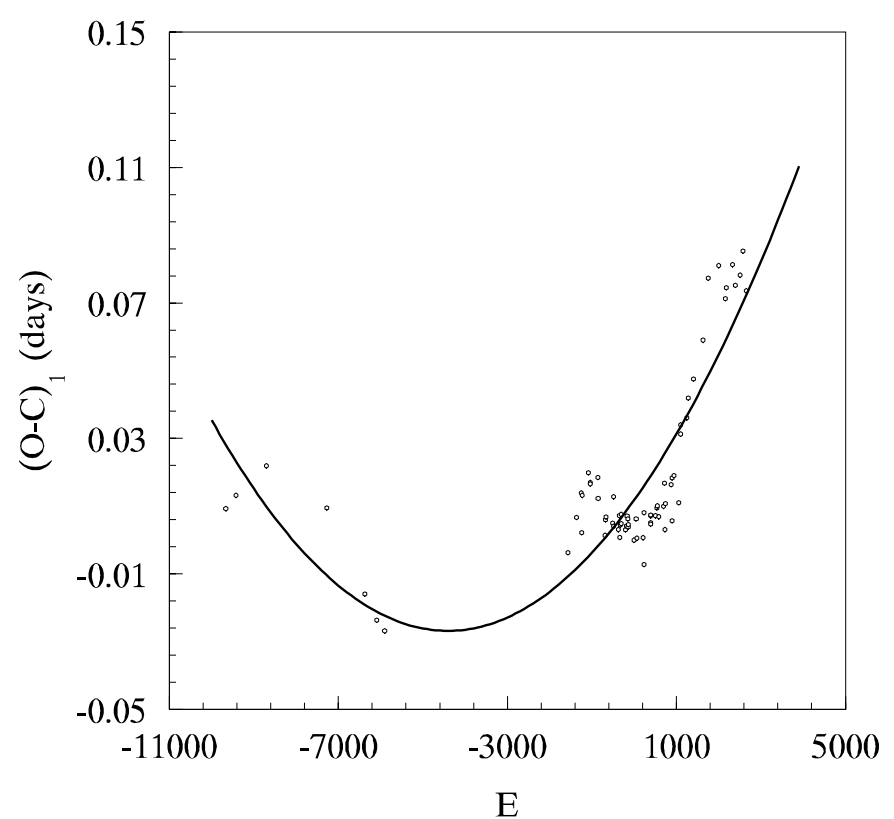

Fig. 6. The O-C diagram of AC Tau. Also given in solid line is its description by a quadratic ephemeris

for the estimation of future times of minima. A continuous period increase of $\mathrm{d} P / \mathrm{d} E=+3.9810^{-9}$ days $/$ cycle $=$ $+7.1110^{-7}$ days/year is calculated which is equivalent to a period increase of $6.1 \dot{\mathrm{s}} /$ century. As in the case of RX Hya, the secular increase only indicates the general trend of the $(\mathrm{O}-\mathrm{C})_{1}$ diagram without describing any particular characteristic.

The $(\mathrm{O}-\mathrm{C})_{2}$ residuals from the quadratic ephemeris (15) are also listed in Table 5 and displayed in Fig. 7. The $(\mathrm{O}-\mathrm{C})_{2}$ values in Fig. 7 clearly suggest a periodic oscillation. With the least-squares method, the following periodic ephemeris is obtained:

$(\mathrm{O}-\mathrm{C})_{2}=0.0069+0.0191 \sin \left(0.0677 \times E+235.7^{\circ}\right)$

which can describe the general trend of the $(\mathrm{O}-\mathrm{C})_{2}$ values wery well (solid line in Fig. 7). This ephemeris tell us a cyclic variation with a period of about $T=29.8$ year and an amplitude of about $A=0$ d 0191 . The cyclic variation of the $\mathrm{O}-\mathrm{C}$ curve indicates that the orbital period varies in a periodic way.

\section{Variation in the orbital period of UW Vir}

Many visual and photographic times of light minimum of UW Vir observed by many amateurs have been compiled at Eclipsing Minimum Database and 4 timings are from Kreiner (1976). In order to analyze the changes in the orbital period of the system, the $(\mathrm{O}-\mathrm{C})_{1}$ residuals of these timings are calculated with the following ephemesris:

$\operatorname{MinI}=2444345.413+1.8107755 \times E$.

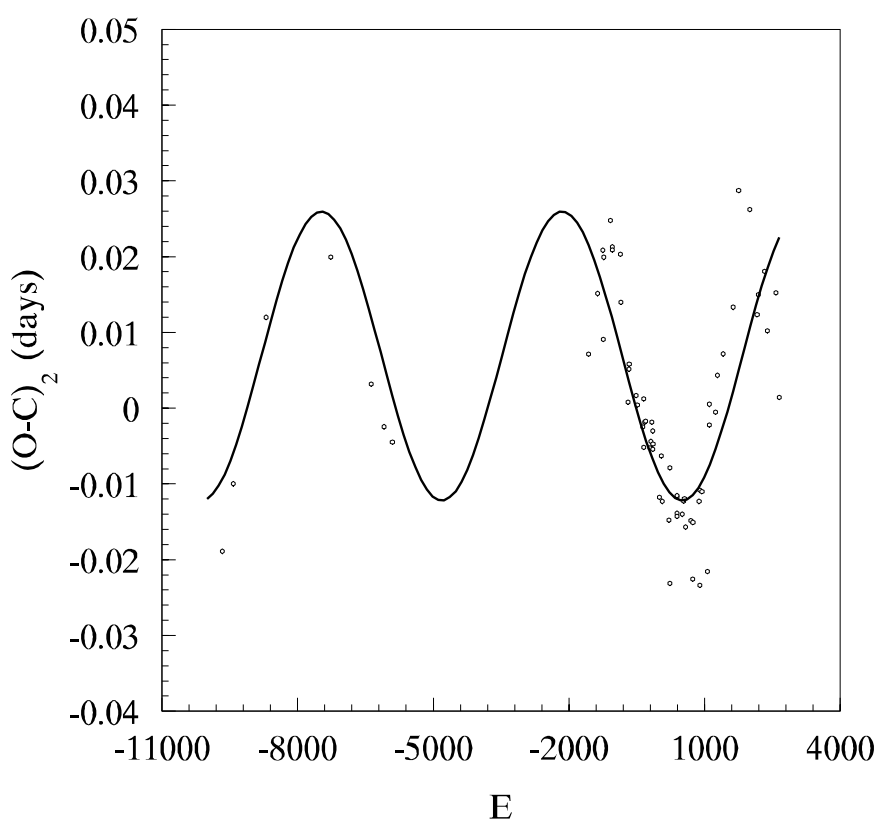

Fig. 7. Residuals of AC Tau from the quadratic ephemeris and their description by a periodic function

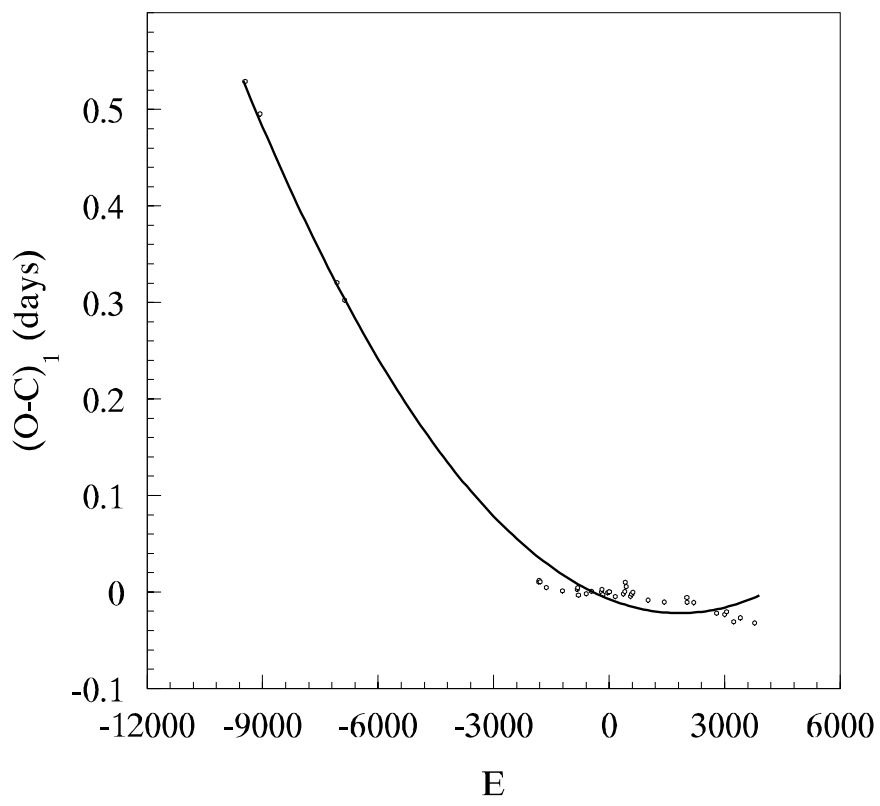

Fig. 8. O-C observations of UW Vir and the description of its general trend (solid line)

These $(\mathrm{O}-\mathrm{C})_{1}$ values are listed in Table 6 and presented graphically against epoch number in Fig. 8. During the calculation, timings with the same epoch have been averaged. Three times of light minimum 2442545.480, 2442545.489 and 2447192.566 with large deviations from the general $\mathrm{O}-\mathrm{C}$ trend formed by other points in Fig. 8 were discarded and not used further in the discussion of the period variation. 
As displayed in Fig. 8, the orbital period of the system is variable, and as in the cases of RX Hya and AC Tau, its variation is in a complex way. Since the general trend of the $(\mathrm{O}-\mathrm{C})_{1}$ diagram may display a roughly parabolic distribution indicating a long-time increase in the orbital period, a second-order least-squares solution of the $(\mathrm{O}-\mathrm{C})_{1}$ values leads to the following ephemeris:

$$
\begin{gathered}
\operatorname{MinI}=2444345.4056+1.81075972 \times E+4.2910^{-9} \times E^{2}(18) \\
\pm 9 \quad \pm 28 \quad \pm 4 .
\end{gathered}
$$

With the coefficient of the square term, a continuous period increase of $\mathrm{d} P / \mathrm{d} E=+8.5810^{-9}$ days $/$ cycle $=$ $+1.7310^{-6}$ days/year is obtained which is equivalent to a period increase of $14.9 \dot{\mathrm{s}}$ /century. As shown in Fig. 8, the secular increase only indicates the general trend of the $(\mathrm{O}-\mathrm{C})_{1}$ diagram without describing any particular characteristics.

The $(\mathrm{O}-\mathrm{C})_{2}$ residuals from the quadratic ephemeris (18) are also listed in Table 6 and displayed in Fig. 9. The $(\mathrm{O}-\mathrm{C})_{2}$ values in Fig. 9 clearly show a cyclic oscillation. The circular orbit of UW Vir (Lucy \& Sweeney 1980) indicates that the periodic oscillation of the $(\mathrm{O}-\mathrm{C})_{2}$ residuals is not caused by apsidal motion. This kind of variation can be explained by the light time effect via the presence of a third body. The sine-like variation of the $(\mathrm{O}-\mathrm{C})_{2}$ curve indicates that the third body is moving in a circular orbit. With the least-squares method, the following periodic ephemeris is obtained:

$(\mathrm{O}-\mathrm{C})_{2}=-0.0155+0.0306 \sin \left(0.0389 \times E+48.67^{\circ}\right)(19)$

which can give a good description to the general trend of the $(\mathrm{O}-\mathrm{C})_{2}$ values (solid line in Fig. 9). This ephemeris describes a periodic variation with a period of about $T=45.9$ year and an amplitude of about $A=0.0306$.

\section{Variation in the orbital period of VV Vul}

VV Vul is also an EA-type eclipsing binary with orbital period 3. 411361 (GCVS, 1985). Halbedel (1984) determined its spectral type to be A2/3V. Up to now, the system is neglected for photometric and spectroscopic studies and no absolute parameter has been published. Whitney (1959) gave 5 visual minima and obtained an ephemeris:

$\operatorname{MinI}=2433266.356+3.41131 \times E$.

After Whitney's (1959) work, 9 times of light minimum published in BBSAG Bulletin have been collected at Eclipsing Minimum Database. With the ephemeris given in the fourth edition of the GCVS,

$\operatorname{MinI}=2445151.570+3.411361 \times E$.

The $\mathrm{O}-\mathrm{C}$ values of the 14 timings are calculated and are listed in the fourth column of Table 7 . The corresponding $\mathrm{O}-\mathrm{C}$ diagram is plotted in Fig. 10.

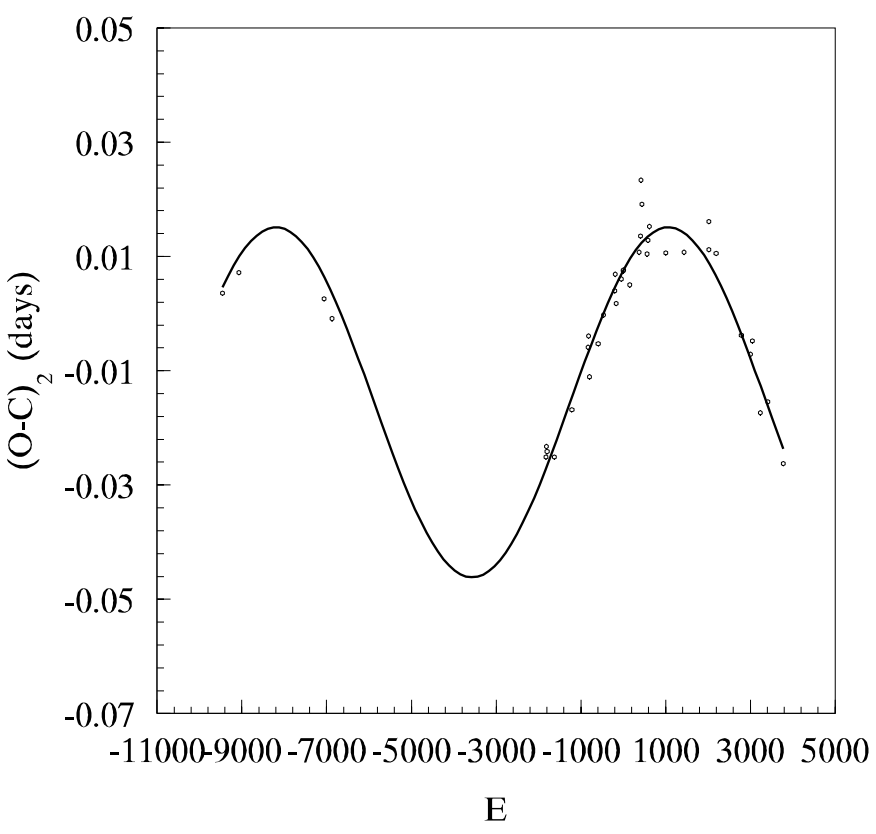

Fig. 9. O-C residuals of UW Vir from the quadratic ephemeris (18)

As shown in Fig. 10, the orbital period of this star is variable. Since no observatins is between $E=-2533$ and $E=-90$, the character of the variation is not clear. Considering a continuous variation in the change of the orbital period, a least square solution leads to the following quadratic ephemeris:

$$
\begin{gathered}
\text { MinI }=2445151.5619+3.41142458 \times E+1.60110^{-8} \times E^{2}(22) \\
\pm 3 \quad \pm 18 \quad \pm 7
\end{gathered}
$$

and to a continuous period increase rate: $\mathrm{d} P / \mathrm{d} E=$ $+3.20210^{-8}$ days $/$ cycle $=+3.4310^{-6}$ days/year which is equivalent to a period increase of $29.6 \dot{\mathrm{s}} /$ century. This ephemeris can be used for the estimation of future times of light minimum.

\section{Discussions and conclusions}

In the previous sections, the changes in the orbital periods of six Algol-type binaries, UW Cyg, RX Hya, AK Ser, AC Tau, UW Vir and VV Vul, are analyzed. It is found that the periods of AK Ser and VV Vul may vary in secular increase, the period of UW Cyg shows a cyclic change. However, the periods of the other three systems, RX Hya, AC Tau and UW Vir vary in complex ways. Periodic variations are found to be superimposed on the long-time increase components.

Algol-type binaries are classified as semi-detached systems whose less massive components are filling their 


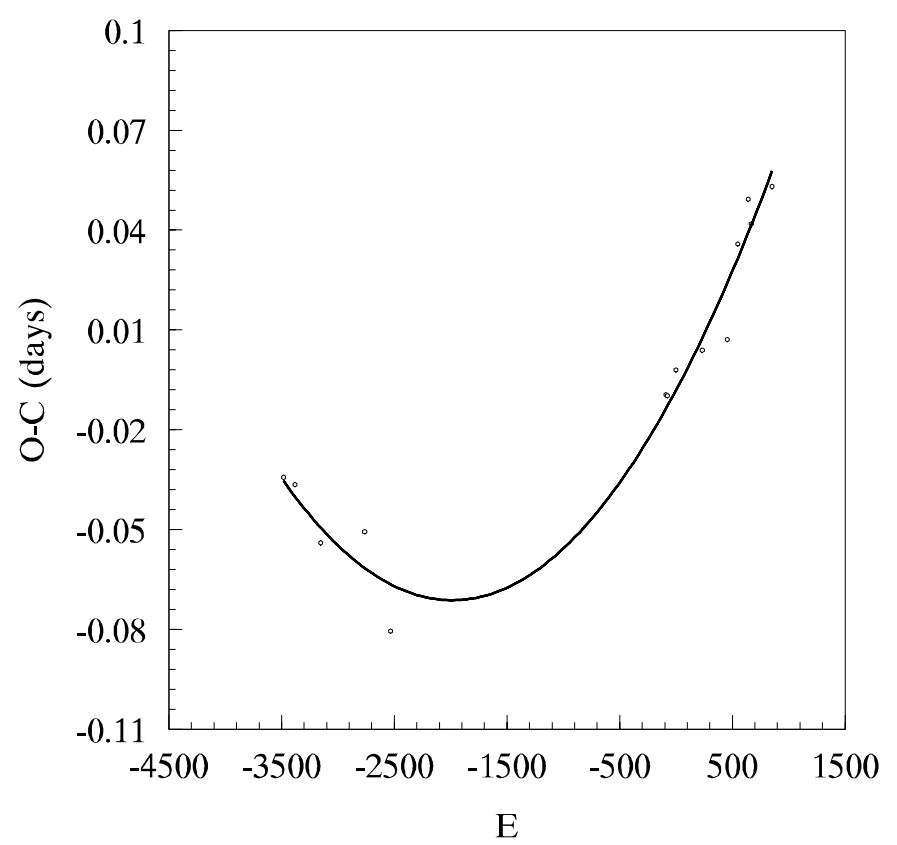

Fig. 10. O-C curve of VV Vul and its description by a quadratic ephemeris

critical Roche Lobe. Assuming the conservation of angular momentum, the orbital periods of Algols should increase during their evolution. The secular increase of the periods in the five system, RX Hya, AK Ser, AC Tau, UW Vir and VV Vul can be explained in terms of the mass transfer from the less massive secondary to the more massive primary. This is consistent with the semi-detached configuration of these systems. If such period changes are due to conservative mass transfer, then by using the parameters listed in Table 1 in the well-known equation:

$\Delta P / P=3\left(M_{1} / M_{2}-1\right) \Delta M_{1} / M_{1}$

the mass transfer rates for theses binaries are computed and listed in Table 8. Since no absolute parameters of VV Vul have been published, its parameters are estimated during the calculation. With the spectral type $\mathrm{A} 2 / 3 \mathrm{~V}$ given by Halbedel (1984), the mass of the primary component is estimated to be $M_{1}=2.5 M_{\odot}$, and considering a typical value of mass ratio $q=0.3$ for Algols, the mass of the secondary is about $0.75 M_{\odot}$.

Apart from 6 photoelectric timings for RX Hya, other times of light minimum analyzed in this paper are visual or photographic, and all times of light minimum for the six systems measured for the primary minimum. This may be caused by the fact that the secondary is more difficult to observe by such methods. The cyclic variations of the $\mathrm{O}-\mathrm{C}$ residuals in the four systems, UW Cyg, RX Hya, AC Tau and UW Vir, are only formed by the times of primary minimum. We do not know whether the secondary timings follow the same trend of oscillation. However,
Table 8. Mass transfer rates for five semi-detached binaries

\begin{tabular}{llll}
\hline Stars & $P($ days $)$ & $\mathrm{d} P / \mathrm{d} t($ days $/$ year $)$ & $\mathrm{d} M / \mathrm{d} t\left(M_{\odot} /\right.$ year $)$ \\
\hline RX Hya & 2.281645 & $+1.0410^{-6}$ & $1.3210^{-7}$ \\
AK Ser & 1.922580 & $+7.9410^{-7}$ & $2.4110^{-7}$ \\
AC Tau & 2.043356 & $+7.1110^{-7}$ & $3.6210^{-7}$ \\
UW Vir & 1.8107755 & $+1.7310^{-6}$ & $1.8110^{-7}$ \\
VV Vul & 3.411361 & $+3.4310^{-6}$ & $3.5910^{-7}$ \\
\hline
\end{tabular}

for RX Hya and UW Vir, Lucy \& Sweeney (1980) have pointed out that their orbits are circular; for the other two, UW Cyg and AC Tau, since their orbital periods are small ( $P<4$ days), a strong mutual tidal interaction between the components may have made their orbits circular. This indicates that the observed cyclic oscillations in $\mathrm{O}-\mathrm{C}$ residuals of the four systems are not caused by apsidal motion. The oscillatory character of the $(\mathrm{O}-\mathrm{C})$ variations in these systems may be result of light time effects due to the presence of additional bodies. As displayed in Figs. 2, 7 and 9, the sine-like $\mathrm{O}-\mathrm{C}$ variations for UW Cyg, AC Tau and UW Vir suggest that their third bodies are moving in circular orbits around the common centre of the gravity of the three bodies. For RX Hya a small eccentricity $\left(e^{\prime}=0.34\right)$ of the orbit of the third body is suggested by the observations. Other orbital parameters are also calculated in Sect. 3.

With the semi-amplitudes of $\mathrm{O}-\mathrm{C}$ oscillations, the value $a_{12}^{\prime} \sin i$ can be computed. Then using the following equation:

$f(m)=\frac{\left(M_{3} \sin i^{\prime}\right)^{3}}{\left(M_{1}+M_{2}+M_{3}\right)^{2}}=\frac{4 \pi^{2}}{G T^{2}} \times\left(a_{12}^{\prime} \sin i^{\prime}\right)^{3}$

we can obtain the mass functions $f(m)$ for the additional bodies. Taking the absolute parameters listed in Table 1, the values of the masses and the orbital dimensions of the third bodies for several different values of $i^{\prime}$ are computed and listed in Table 9. If we assume that the orbital inclination is perpendicular to the visual line (i.e., $i^{\prime}=90^{\circ}$ ), the values of the masses of the additional bodies are computed to be $m_{3}=1.12,1.50,0.75$ and $0.87 M_{\odot}$ for UW Cyg, RX Hya, AC Tau and UW Vir. At this case the orbital radii of the third bodies should be: $a_{3}=13.64,16.83$, 10.77, 13.40 AU respectively.

As listed in Table 9, for RX Hya, the minimum mass of the third body is $m_{3}=1.50 M_{\odot}$ which is smaller than the corresponding value $2.25 M_{\odot}$ derived by Vyas \& Abhyankar (1989) from their orbital period analysis. If the third component is main sequence star as the components of RX Hya, the computed mass will correspond to a spectral type G1-3 which is nearly close to the spectral type of this system (F1). This indicates that the spectral line of the third body should be visible in the spectrum and the third star should contribute to the total 
Table 9. The values of the masses and the orbit radii of the third bodies in UW Cyg, RX Hya, AC Tau and UW Vir

\begin{tabular}{llllll}
\hline & UW Cyg & RX Hya & AC Tau & UW Vir & Units \\
\hline$A$ & 0.0383 & 0.0431 & 0.0191 & 0.0306 & days \\
$T$ & 49.3 & 54.3 & 29.8 & 45.9 & years \\
$a_{12}^{\prime} \sin i^{\prime}$ & 6.64 & 7.47 & 3.31 & 5.30 & $\mathrm{AU}$ \\
$f(m)$ & $1.2110^{-1}$ & $1.4110^{-1}$ & $4.0910^{-2}$ & $7.0710^{-2}$ & $M_{\odot}$ \\
$m_{3}\left(i^{\prime}=90^{\circ}\right)$ & 1.12 & 1.50 & 0.75 & 0.87 & $M_{\odot}$ \\
$m_{3}\left(i^{\prime}=70^{\circ}\right)$ & 1.21 & 1.62 & 0.80 & 0.94 & $M_{\odot}$ \\
$m_{3}\left(i^{\prime}=50^{\circ}\right)$ & 1.60 & 2.12 & 1.03 & 1.23 & $M_{\odot}$ \\
$m_{3}\left(i^{\prime}=30^{\circ}\right)$ & 3.00 & 3.92 & 1.81 & 2.23 & $M_{\odot}$ \\
$m_{3}\left(i^{\prime}=10^{\circ}\right)$ & 27.0 & 32.8 & 11.5 & 17.2 & $M_{\odot}$ \\
$a_{3}\left(i^{\prime}=90^{\circ}\right)$ & 13.64 & 16.83 & 10.77 & 13.40 & $\mathrm{AU}$ \\
$a_{3}\left(i^{\prime}=70^{\circ}\right)$ & 13.43 & 16.59 & 10.74 & 13.20 & $\mathrm{AU}$ \\
$a_{3}\left(i^{\prime}=50^{\circ}\right)$ & 12.46 & 15.55 & 10.24 & 12.37 & $\mathrm{AU}$ \\
$a_{3}\left(i^{\prime}=30^{\circ}\right)$ & 10.18 & 12.88 & 8.924 & 10.46 & $\mathrm{AU}$ \\
$a_{3}\left(i^{\prime}=10^{\circ}\right)$ & 3.257 & 4.433 & 4.044 & 3.904 & $\mathrm{AU}$ \\
\hline
\end{tabular}

luminosity of the system. In the absence of such evidences, the presence of the third body needs further study.

For UW Cyg, the minimum mass of the third body $\left(m_{3}=1.12 M_{\odot}\right)$ corresponds to a spectral type G5-7 which is also close to the spectral type of this system (F0). From the spectroscopic and photoelectric observations, we should be able to find the presence of the third body. In the cases of AC Tau and UW Vir, since the values $0.75 M_{\odot}$ and $0.87 M_{\odot}$ are also the minimum masses of the third bodies, there is a possibility to see their spectral lines in the spectra. However, to the best of my knowledge, no spectroscopic and photometric studies of the three systems, UW Cyg, AC Tau and UW Vir, have been published. This emphasizes the urgent need of future photometric and particularly spectroscopic observations to decide on the hypothetical third bodies.

Acknowledgements. Many thanks are given to Prof. F. van't Veer for his useful comments and improvements of the author's English writing. The author also thanks the supports from the Chinese Natural Science Foundation and from the 973 scheme.

\section{References}

Brancewicz H.K., Dworak T.Z., 1980, AcA 30, 501 Dugan R.S., 1933, Princeton Cont., No. 14, 1

Halbedel E.M., 1984, IBVS 2594

Kholopov P.N., 1985, The fourth edition of the General Catalogue of Variable Stars

Kopal Z., 1959, Close Binary Systems. Chapman \& Hall, Ltd., London, p. 495

Kreiner J.M., 1976, AcA 26, 341

Lucy L.B., Sweeney M.A., 1971, AJ 76, 544

Maggini M., 1921, Men. Soc. Astron. It., n.s. 2, 293

Mallama A.D., 1980, ApJS 44, 241

Pickering E.C., 1907, Harvard Circ., No. 133 (Astron. Nach. (1907) 176, 297 (4218))

Struve O., 1946, ApJ 104, 253

Szczepanowska A., 1959, AcA 9, 46

Vyas M.L., Abhyankar K.D., 1989, A\&AS 81, 67

Whitney B.S., 1959, AJ 64, 258

Wood B.D., Forbes J.E., 1963, AJ 68, 257

Zinner E., 1922, Astron. Abh. 4, C3(3) 